\title{
CASIMIR ENERGY IN NON-COVARIANT GAUGES
}

Giampiero Esposito $^{1,2}$, Alexander Yu. Kamenshchik ${ }^{3,4}$ and K. Kirsten ${ }^{5}$

${ }^{1}$ Istituto Nazionale di Fisica Nucleare, Sezione di Napoli, Complesso Universitario di Monte S. Angelo, Via Cintia, Edificio N', 80126 Napoli, Italy

${ }^{2}$ Università degli Studi di Napoli Federico II, Dipartimento di Scienze Fisiche, Complesso Universitario di Monte S. Angelo, Via Cintia, Edificio N', 80126 Napoli, Italy

${ }^{3}$ L.D. Landau Institute for Theoretical Physics, Russian Academy of Sciences, Kosygina Str. 2, Moscow 117334, Russia

${ }^{4}$ Landau Network-Centro Volta, Villa Olmo, Via Cantoni 1, 22100 Como, Italy

${ }^{5}$ The University of Manchester, Department of Physics and Astronomy, Theory Group, Schuster Laboratory, Oxford Road, Manchester M13 9PL, England

\begin{abstract}
The zero-point energy of a conducting spherical shell is studied by imposing the axial gauge via path-integral methods, with boundary conditions on the electromagnetic potential and ghost fields. The coupled modes are then found to be the temporal and longitudinal modes for the Maxwell field. The resulting system can be decoupled by studying a fourth-order differential equation with boundary conditions on longitudinal modes and their second derivatives. Complete agreement is found with a previous pathintegral analysis in the Lorenz gauge, and with Boyer's value. This investigation leads to a better understanding of how gauge independence is achieved in quantum field theory on backgrounds with boundary.
\end{abstract}


The quantization programme of fundamental interactions in non-covariant gauges has been always of great importance, as is well known from the work in Refs. [1,2]. On the other hand, recent progress in the measurement of Casimir energies [3-6] motivates new theoretical efforts, including the comparison of covariant and non-covariant gauges for the evaluation of Casimir energies.

We here rely entirely on the path-integral analysis of Ref. [7], and avoid review for length reasons. Recall therefore that, in the case of a spherical shell of radius $R$, the axial gauge forces normal modes of the potential to vanish everywhere, jointly with ghost modes. One is then left with transverse modes $T_{l}(r)=\sqrt{\pi / 2} \sqrt{r} I_{l+1 / 2}(M r)$ which obey homogeneous Dirichlet conditions at $r=R$, while temporal and longitudinal modes (denoted by $a_{l}$ and $c_{l}$, respectively), obey the coupled system [7]

$$
\begin{gathered}
{\left[\frac{d^{2}}{d r^{2}}+\frac{2}{r} \frac{d}{d r}-\frac{l(l+1)}{r^{2}}\right] a_{l}-\frac{M l(l+1)}{r^{2}} c_{l}=0,} \\
{\left[\frac{d^{2}}{d r^{2}}-M^{2}\right] c_{l}-M a_{l}=0 .}
\end{gathered}
$$

First, we now point out that this system can be decoupled if $a_{l}$ is expressed from Eq. (2) and the result is inserted into Eq. (1). On setting $y \equiv M r$, this leads to the fourth-order equation

$$
\left[\frac{d^{4}}{d y^{4}}+\frac{2}{y} \frac{d^{3}}{d y^{3}}-\left(1+\frac{l(l+1)}{y^{2}}\right) \frac{d^{2}}{d y^{2}}-\frac{2}{y} \frac{d}{d y}\right] c_{l}(y)=0
$$

Moreover, we know from Ref. [7] that, at $r=R$, both $a_{l}$ and $c_{l}$ should vanish. By virtue of Eq. (2) this implies that, at $r=R$, both $c_{l}$ and its second derivative should vanish. The origin $r=0$ is a regular singular point of our boundary-value problem, and hence we require that both $a_{l}$ and $c_{l}$ should vanish therein. By virtue of Eq. (2), this implies that also the second derivative of $c_{l}$ with respect to $y$ vanishes at $y=0$. Now we set $F_{l}(y) \equiv c_{l}^{\prime}(y)$, and cast Eq. (3) in the form

$$
\frac{1}{y^{2}} \frac{d}{d y}\left[y^{2}\left(\frac{d^{2}}{d y^{2}}-\left(1+\frac{l(l+1)}{y^{2}}\right)\right) F_{l}(y)\right]=0
$$


On denoting by $C$ a constant, the desired $F_{l}(y)$ solves therefore the inhomogeneous equation

$$
\left[\frac{d^{2}}{d y^{2}}-\left(1+\frac{l(l+1)}{y^{2}}\right)\right] F_{l}(y)=\frac{C}{y^{2}},
$$

whose regular solution reads ( $d$ being a constant)

$$
F_{l}(y)=C F_{s p}(y)+d \sqrt{\pi / 2} \sqrt{y} I_{l+1 / 2}(y)
$$

where $F_{s p}$ is chosen to be a particular solution of Eq. (5) with $C=1$ whose first derivative vanishes at 0 and at $b \equiv M R$. The vanishing of $c_{l}$ at the boundary fixes the relation between $C$ and $d$, i.e.

$$
C=-d \frac{\int_{0}^{b} d z \sqrt{\pi / 2} \sqrt{z} I_{l+1 / 2}(z)}{\int_{0}^{b} d z F_{s p}(z)} .
$$

Eventually, the vanishing of $c_{l}^{\prime \prime}$ at the boundary leads to

$$
I_{l+1 / 2}^{\prime}+\frac{1}{2 b} I_{l+1 / 2}(b)=0
$$

because, by construction, $C F_{s p}$ is a particular solution of Eq. (5) whose first derivative vanishes at the boundary. Equation (8), jointly with the vanishing at the boundary of transverse modes, yields the same set of eigenvalue conditions, with the same degeneracies, found for the interior problem in the Lorenz gauge in Ref. [7]. Thus, complete agreement [8] with Boyer's value [9] for the Casimir energy is recovered.

Although non-covariant gauges break relativistic covariance, they make it possible to decouple Faddeev-Popov ghosts from the gauge field. Thus, ghost diagrams do not contribute to cross-sections and need not be evaluated, and this property has been regarded as the main advantage of non-covariant gauges [2]. In the case of Casimir energies, the ghost field is forced to vanish everywhere by virtue of the boundary conditions appropriate for the axial gauge [7], and the analysis of temporal and longitudinal modes, although rather involved, has been proved to lead to the same Casimir energy [9] for the interior problem in the Lorenz gauge [7]. The particular solution of the inhomogeneous equation (5) plays a non-trivial role in ensuring that, despite some technical points, the resulting Casimir 
energy is the same as in the Lorenz gauge, hence proving explicitly the equivalence of a covariant and a non-covariant gauge for a conducting spherical shell. A better theoretical understanding of gauge independence in quantum field theory has been therefore gained, after the encouraging experimental progress of recent years in the measurement of Casimir forces $[3-6]$.

Acknowledgments. Klaus Kirsten thanks Stuart Dowker for interesting discussions. The work of Klaus Kirsten has been supported by EPSRC, grant no GR/M08714.

\section{References}

[1] G. Leibbrandt, Rev. Mod. Phys. 59, 1067 (1987).

[2] G. Leibbrandt, Noncovariant Gauges (World Scientific, Singapore, 1994).

[3] S. K. Lamoreaux, Phys. Rev. Lett. 78, 5 (1997).

[4] U. Mohideen and A. Roy, Phys. Rev. Lett. 81, 4549 (1998).

[5] A. Roy and U. Mohideen, Phys. Rev. Lett. 82, 4380 (1999).

[6] A. Roy, C. Y. Liu and U. Mohideen, Phys. Rev. D 60, 111101 (1999).

[7] G. Esposito, A. Yu. Kamenshchik and K. Kirsten, Int. J. Mod. Phys. A 14, 281 (1999).

[8] G. Esposito, A. Yu. Kamenshchik and K. Kirsten, "Casimir Energy in the Axial Gauge" (DSF preprint 2000/21, hep-th 0006220).

[9] T. H. Boyer, Phys. Rev. 174, 1764 (1968). 